\title{
Research article \\ Effect of a mannose to the interactions between Naegleria fowleri and pathogenic bacteria
}

\author{
Suk-Yul Jung \\ Associate Professor, Department of Biomedical Laboratory Science, Molecular Diagnostics Research Institute, Namseoul \\ University, Cheonan 31020, Republic of Korea
}

(Received: May $2021 \quad$ Revised: June $2021 \quad$ Accepted: July 2021)

Corresponding author: Suk-Yul Jung. Email: syjung@nsu.ac.kr

\begin{abstract}
Introduction and Aim: In this study, the interaction between pathogenic Naegleria fowleri and pathogenic bacteria such as methicillin-resistant Staphylococcus aureus (MRSA), Enterococcus faecalis and Salmonella typhi was analyzed by a monosaccharide of mannose. Moreover, since the form of $N$. fowleri was found in diseases as cysts, the interaction between cysts and bacteria was analyzed.

Materials and Methods: In order to analyze the role of a monosaccharide called mannose in bacterial interaction, the analysis of bacterial association, invasion, and survival for amoeba treated with mannose was performed. $N$. fowleri trophozoites or cysts were pre-treated with a mannose at a concentration of 10, 50 and $100 \mathrm{mM}$ for $1 \mathrm{hr}$ at $37^{\circ} \mathrm{C}$.

Results: The MRSA association was hardly suppressed until the concentration of mannose was $50 \mathrm{mM}$, but its association was reduced by about $168 \%$ to $N$. fowleri trophozoites by $100 \mathrm{mM}$ mannose. Compared to the results for MRSA, the association of $E$. faecalis had little effect by mannose. Very interestingly, although $S$. typhi showed much higher invasion than the above MRSA and E. faecalis, it did not survive at all within $N$. fowleri trophozoites. Ten $\mathrm{mM}$ mannose showed a nearly similar $1 \%$ association with $N$. fowleri cyst treated, but not with $50 \mathrm{mM}$ and $100 \mathrm{mM}$ mannose treated $N$. fowleri cyst at all.
\end{abstract}

Conclusion: The association and invasion of $S$. typhi was highest for $N$. fowleri trophozoites and cysts, but the three bacteria did not survive in $N$. fowleri trophozoites and cysts.

Keywords: Naegleria fowleri; interaction; MRSA; Enterococcus faecalis; Salmonella typhi.

\section{INTRODUCTION}

$N$ aegleria fowleri, a brain-eating amoeba, is a protozoan that induces primary amoebic encephalitis in the cerebrum through the epithelial cells of the nose in humans and experimental animals and causes a very high mortality rate $(1,2) . N$. fowleri, such as Acanthamoeba, can help bacteria such as Legionella pneumophila to grow in the cytoplasm. However, it has been reported that environmental microorganisms exist in the cytoplasm of the nonpathogenic Naegleria strain but reports on the exact life cycle or survival of the microorganism against the pathogenic amoeba, $N$. fowleri, are insufficient $(3,4)$.

When $N$. fowleri destroys host cells, it binds to the host cells. $N$. fowleri destroys the host cell by the proteolytic enzyme secreted by $N$. fowleri, to proliferate and invade in central nervous system (CNS) $(2,5)$ and can express amoeba structures such amoebastome that can be physically contacted to host cells $(6,7)$. There are certain factors that trigger the adhesion of organisms, such as pore-forming proteins, the presence of carbohydrate residues on the outer surface of the plasma membrane, and the presence of terminal -L-Fucose and -D-glucose in glycol conjugates $(2,8)$.

Acanthamoeba, a free-living amoeba similar to Naegleria, is known to interact with several bacteria such as Escherichia coli K1 (a bacterium that causes meningitis) (9-11) and L. pneumophila (a bacterium that causes Legionella disease) (12). These bacteria can act as food for Acanthamoeba and contain some kind of polysaccharide layer (e.g., cell wall). The adhesion is a very important first step in amoeba's attacking host cells. It has been reported that non-pathogenic amoebae are less capable of binding to host cells (13). Contactdependent interactions of these monosaccharidebinding proteins or monosaccharides itself may clarify the pathogenicity or infectivity of the free-living amoeba $(9,11)$. In the previous report, the mannosebinding protein of $A$. culbertsoni could act as a very important factor in the interaction between amoeba and target cells (14).

The association between $A$. culbertsoni pretreated with polyclonal serum for a mannose-binding protein (MBP) and pathogenic bacteria such as E. coli $\mathrm{O} 157: \mathrm{H7}$, Staphylococcus aureus and Bacillus subtilis was found to be reduced by about 35 to $40 \%$, and monoclonal antibodies also showed similar results (15). To understand the interaction between bacteria and freeliving amoeba in this way, it is necessary not only to study the contact, but also to analyze the invasion of bacteria and the survival of bacteria within the amoeba in more detail. Therefore, in this study, the interaction between pathogenic $N$. fowleri and pathogenic bacteria such as methicillin-resistant $S$. aureus (MRSA), Enterococcus faecalis and Salmonella typhi was 
analyzed. In addition, since mannose sugar acted as a very important ligand in the Acanthamoeba study, it was tried to determine what changes occurred in the interaction with bacteria by treating mannose sugar in $N$. fowleri. Moreover, since the form of $N$. fowleri was found in diseases as cysts, the interaction between cysts and bacteria was analyzed.

\section{MATERIALS AND METHODS}

\section{Culture of $N$. fowleri trophozoites and inducement of N. fowleri cysts}

Unless otherwise stated, all reagents and laboratory materials were purchased from Sigma (Seoul, Korea). $N$. fowleri (Carter NF69 strain, ATCC No. 30215) trophozoites were aseptically cultured at $37^{\circ} \mathrm{C}$ in Nelson's medium (1.7 g neutralized liver digest (Oxoid, Basingstoke, England), $1.7 \mathrm{~g}$ glucose, $4 \mathrm{mg}$ $\mathrm{MgSO}_{4} \cdot 7 \mathrm{H}_{2} \mathrm{O}, 4 \mathrm{mg} \mathrm{CaCl} \cdot 2 \mathrm{H}_{2} \mathrm{O}, 142 \mathrm{mg} \mathrm{Na} 2 \mathrm{HPO}_{4}$, $136 \mathrm{mg} \mathrm{KH}{ }_{2} \mathrm{PO}_{4}, 120 \mathrm{mg} \mathrm{NaCl}, 10 \%$ fetal bovine serum (ThermoFisher Scientific, Seoul, Korea) (16). The medium was exchanged when the trophozoites became $70 \%$ confluene by periodically observing it under an inverted microscope. For the analysis of bacterial interactions against $N$. fowleri cysts, cysts were induced from $N$. fowleri trophozoites. This method was previously performed by referring to the method used for the cyst induction of Acanthamoeba (11). N. fowleri trophozoites were transferred to a 3\% non-nutrient agar plate (Oxoid limited, Seoul, Korea), and the plate was incubated for up to 4 days at $30^{\circ} \mathrm{C}$. Cysts forming groups were periodically observed under a bright microscope. After addition of phosphate-buffered saline (PBS), the surface of the agar was gently scraped off using a cell scraper to finally confirm the formation of cysts.

\section{Culture of bacteria}

Three pathogenic bacteria such as MRSA (ATCC No. BAA-1769), E. faecalis (KCTC No. 5290) and S. typhi (ATCC No. 19430) were applied in the interaction with $N$. fowleri trophozoites and cysts.

The number of bacterial colonies was calculated for the interaction analysis. Therefore, all bacteria were cultured using tryptic soy agar (TSA, MB cell, Korea), which was a generally used enrichment medium instead of the bacterial selection medium. Bacteria were cultured at $37^{\circ} \mathrm{C}$, and the bacteria were once again confirmed through Gram staining (17). Since bacterial colonies were accurately calculated and added to the amoeba above, the following experiment was performed by adjusting the McFaland turbidity of 0.5 representing $0.5 \times 10^{3}$ to $1.5 \times 10^{6}$ colony forming units $(\mathrm{cfu}) / \mathrm{mL}$.

\section{Bacterial association, invasion assay and survival assay}

It is known that $N$. fowleri is capable of predating and binding bacteria but reports of how bacteria interact with $N$. fowleri are still weak. In previous studies on $A$. culbertsoni, a free-living amoeba, the mannose binding protein (MBP) played a very important role in interactions with host cells. Therefore, in this study, to analyze the role of a monosaccharide called mannose in bacterial interaction, the analysis of bacterial association, invasion, and survival for amoeba treated with mannose was performed. All experimental methods have been changed slightly from a previous report (11). N. fowleri trophozoites or cysts were pretreated with a mannose at a concentration of 10,50 and $100 \mathrm{mM}$ for $1 \mathrm{hr}$ at $37^{\circ} \mathrm{C}$ and the washed two times with PBS. All bacteria were added to $N$. fowleri trophozoites or cysts by adjusting to a $0.5 \mathrm{McF}$ arland index. Briefly, $N$. fowleri trophozoites or cysts were incubated in a 24well culture plate with Nelson medium. N. fowleri trophozoites or cysts pre-treated with mannose was incubated with bacteria ( $2 \times 10^{5} \mathrm{cfu} / 0.5 \mathrm{ml}$ of PBS) at room temperature (RT) for $1 \mathrm{hr}$. After washing three times with PBS, SDS (final concentration $0.5 \%$ ) was added to each well for $30 \mathrm{~min}$ to destroy N. fowleri, and the number of bacterial colonies produced was calculated by incubating in a TSA plate for one day. The result obtained is called bacterial association and the calculation method is as follows: recovered bacteria (cfu) / total bacteria $(\mathrm{cfu}) \times 100=\%$ of bacteria associated with $N$. fowleri. Since the bacteria would associate with $N$. fowleri cell wall and then invade, the invasion assay proceeded as follows. After the association analysis, gentamicin antibiotics was added for 45 minutes (100 $\mu \mathrm{g} / \mathrm{ml}$, final concentration) to remove bacteria not associated with $N$. fowleri. The result obtained was expressed as the number of colonies of the recovered bacteria relative to the total colonies of the invading bacteria as shown in the above association result. The plate after the invasion assay was placed at $37^{\circ} \mathrm{C}$ for 24 $\mathrm{hr}$ to see how much of the invading bacteria survived within N. fowleri. Thereafter, the supernatant was removed, and colonies of bacteria cultured in TSA for one day were calculated to calculate the survival of bacteria. The calculation method evaluated the number of bacteria recovered as described above.

\section{Statistical analysis}

To determine the statistical significance of the resulting values of the interaction, Student two-sample t test was performed. If $\mathrm{P}<0.05$, it was determined to show statistical significance.

\section{RESULTS}

\section{Effect of mannose on $N$. fowleri trophozoites in the interaction with MRAS}

In the study of Acanthamoeba, MBP was known to play an important role in the contact-dependent pathway. Therefore, in this experiment, it was analyzed how mannose binding to MBP affected the interaction between $N$. fowleri trophozoites and MRSA, namely, bacterial association, invasion, and survival. As shown in the results of Fig. 1, it was observed that $N$. fowleri trophozoites, treated with a high concentration of 100 $\mathrm{mM}$ mannose, inhibited the interaction with bacteria. 

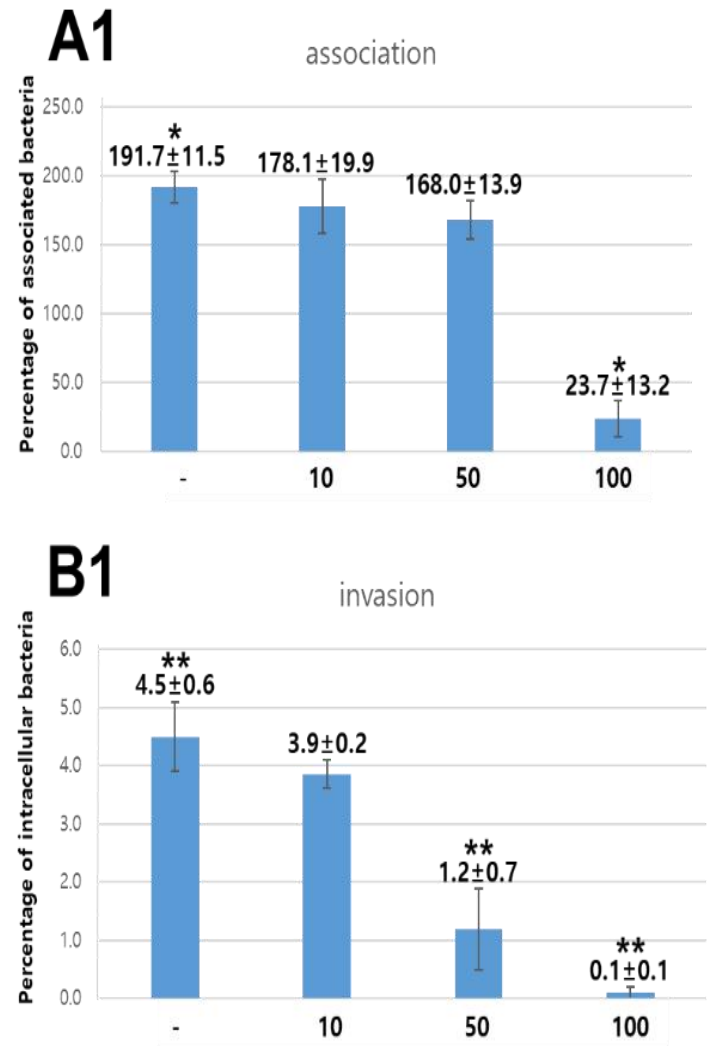

\section{C1}

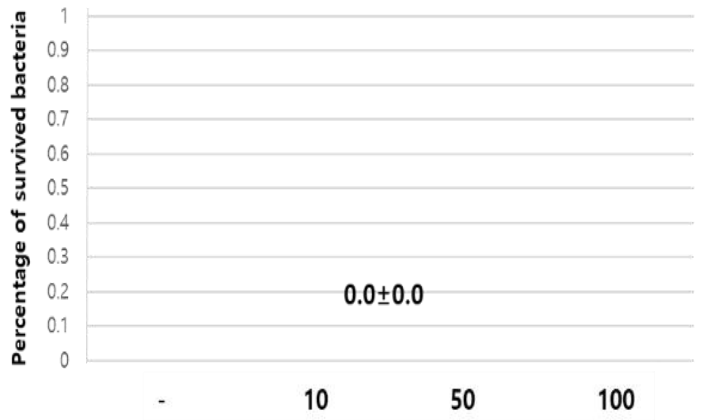

A2

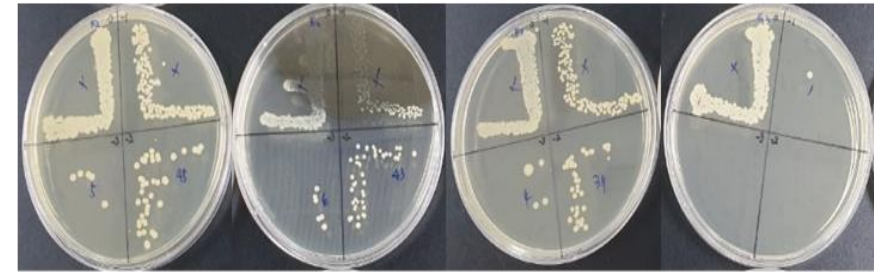

B2

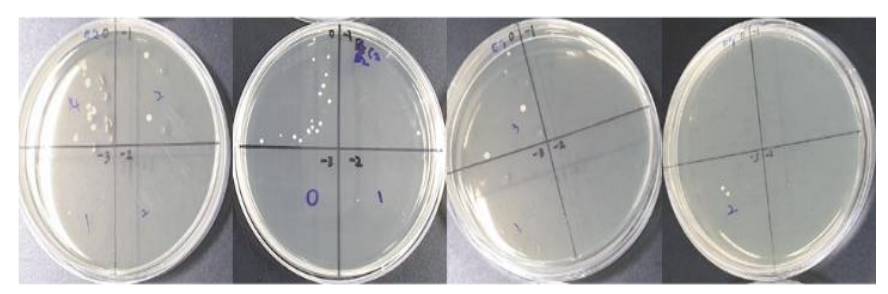

C2

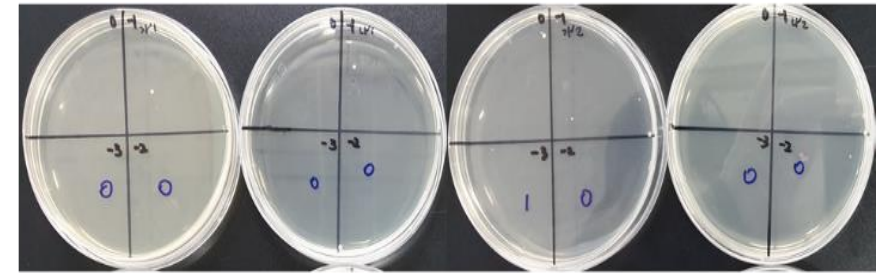

Fig. 1: MRSA association, invasion, and survival to $N$. fowleri trophozoites. A1, B1 and C1 represented the percentage of MRSA association, invasion, and survival of the bacteria, respectively, and A2, B2 and C2 represented the plates for A1, B1 and $\mathrm{C} 1$, respectively. The number on the $\mathrm{X}$-axis indicated the $\mathrm{mM}$ concentration of mannose in $\mathrm{A} 1, \mathrm{~B} 1$ and $\mathrm{C} 1$. This experiment was performed in triplicate wells with three times, and data were indicated by standard deviation (SD) value.

In a more detailed analysis, the results of bacterial association showed that the bacterial association was hardly suppressed until the concentration of mannose was $50 \mathrm{mM}$, but the association of bacteria was reduced by about $168 \%$ with respect to untreated $N$. fowleri trophozoites by $100 \mathrm{mM}$ mannose (Fig. 1 A1, B1) (P $<0.05$ ). On the other hand, there was not much difference between the results of mannose on the invasion results compared to the results of bacterial association (Fig. 1 A2, B2). The invasion of MRSA was reduced by about $4.3 \%$ and $4.4 \%$ for untreated $N$. fowleri trophozoites by $50 \mathrm{mM}$ and $100 \mathrm{mM}$ mannose, respectively. However, assuming that the invasion of MRSA without mannose treatment was $100 \%$, its invasion was decreased by about $73.4 \%$ by $50 \mathrm{mM}$ mannose and about $97.8 \%$ by $100 \mathrm{mM}$ mannose ( $\mathrm{P}$ $<0.05)$. Interestingly, MRSA did not survive in $N$. fowleri trophozoites at all compared to N. fowleri trophozoites treated with mannose from the control group (Fig. 1 A3, B3).

\section{Effect of mannose on $\boldsymbol{N}$. fowleri trophozoites in the interaction with $E$. faecalis}

E. faecalis belonging to Group D Streptococcus, is a bacterium that lives in the human gastrointestinal tract. In order to analyze the interaction between $E$. faecalis and mannose treated $N$. fowleri trophozoites, it was carried out by the same method as MRSA mentioned above (Fig. 2). 

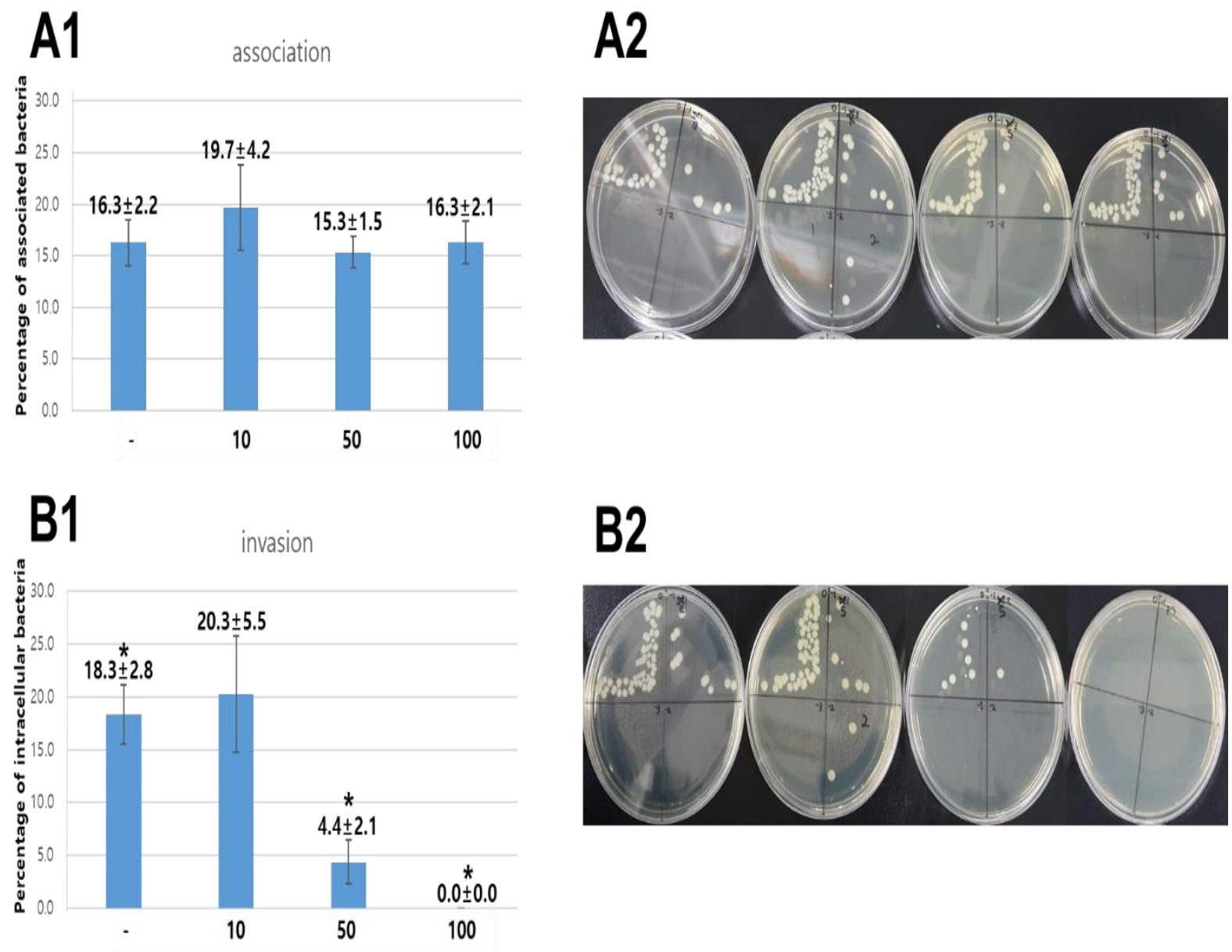

B2
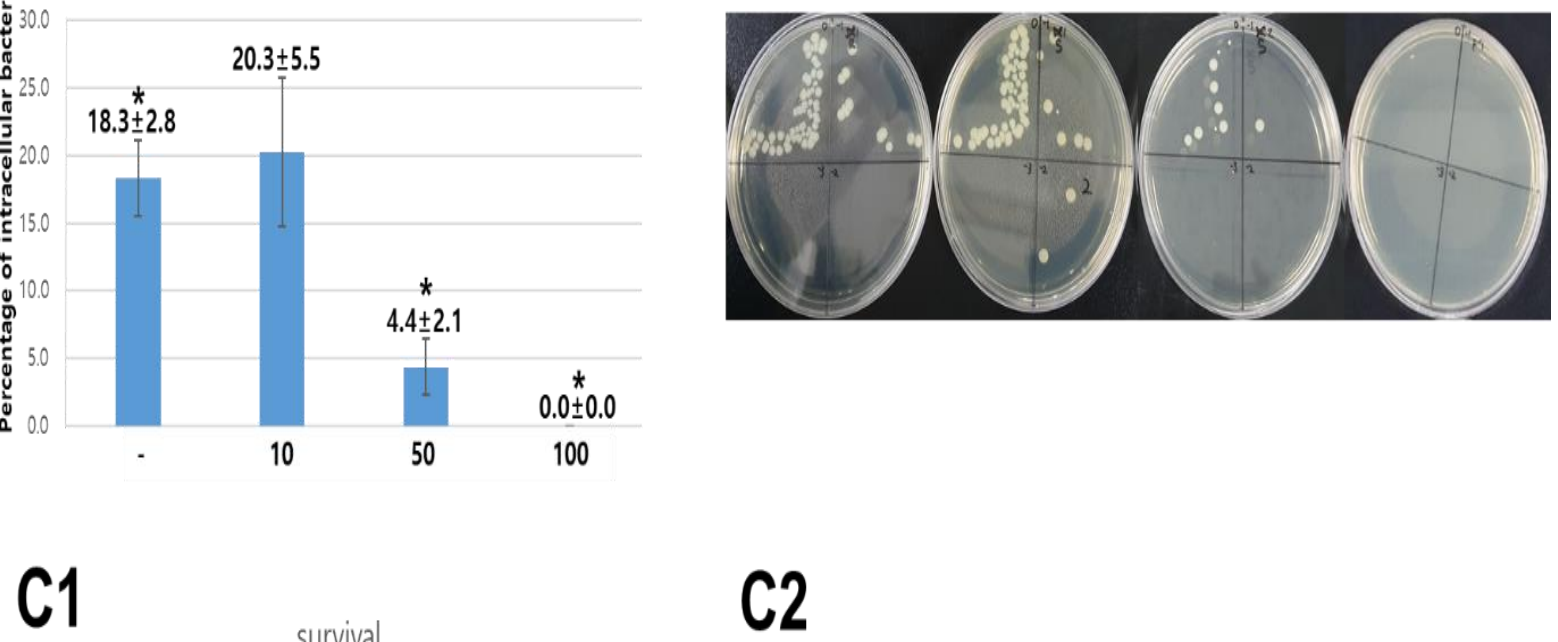

survival

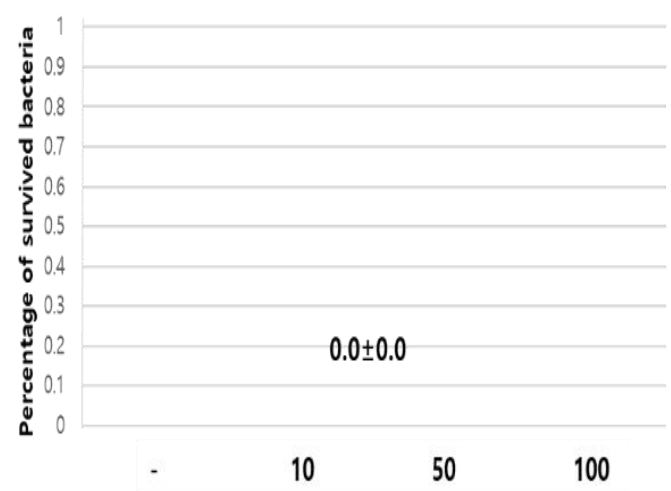

C2

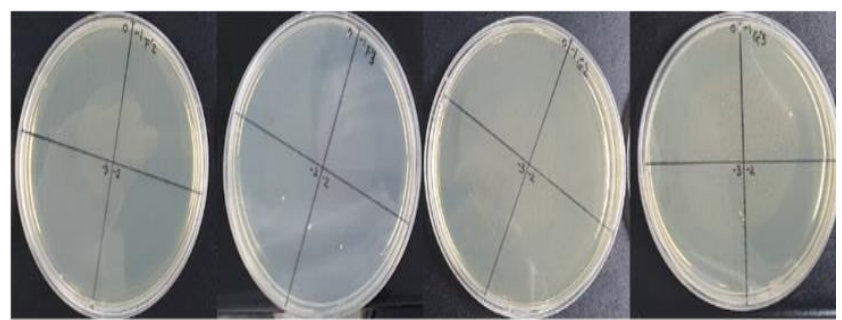

Fig. 2: E. faecalis association, invasion, and survival to $N$. fowleri trophozoites. A1, B1 and C1 represented the percentage of E. faecalis association, invasion, and survival of the bacteria, respectively, and A2, B2 and C2 represented the plates for A1, B1 and $\mathrm{C} 1$, respectively. The number on the $\mathrm{X}$-axis indicated the $\mathrm{mM}$ concentration of mannose in $\mathrm{A} 1, \mathrm{~B} 1$ and $\mathrm{C} 1$. This experiment was performed in triplicate wells with three times, and data were indicated by SD value.

Compared to the results for MRSA, the association of $E$. faecalis had little effect by mannose (Fig. 2 A1, B1). However, the invasion of $E$. faecalis was reduced by about $14 \%$ with $50 \mathrm{mM}$ mannose than when mannose was not treated (Fig. 2 A2, B2) $(\mathrm{P}<0.05)$. In addition, invasion of E. faecalis to $N$. fowleri trophozoites pretreated with $100 \mathrm{mM}$ mannose did not occur at all (P $<0.05$ ). E. faecalis did not survive in $N$. fowleri trophozoites at all, very similar to the results of MRSA (Fig. 2 A3, B3).

\section{Effect of mannose on $N$. fowleri trophozoites in the interaction with $S$. typhi}

There was no statistically significant difference compared to the association between MRSA and $E$. faecalis and the results of invasion, but the association and invasion of $S$. typhi were much higher than those of the other two bacteria such as MRSA and E. faecalis (Fig. 3). 


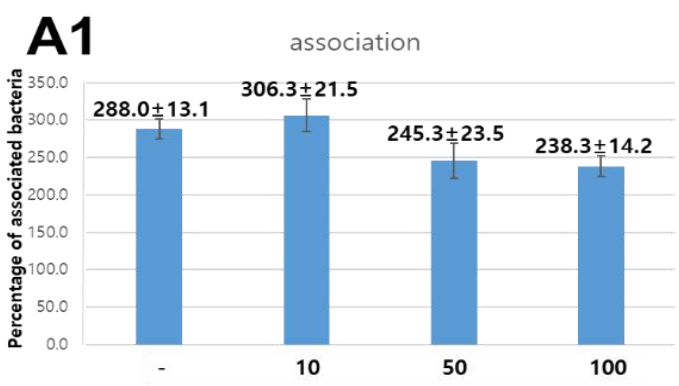

A2

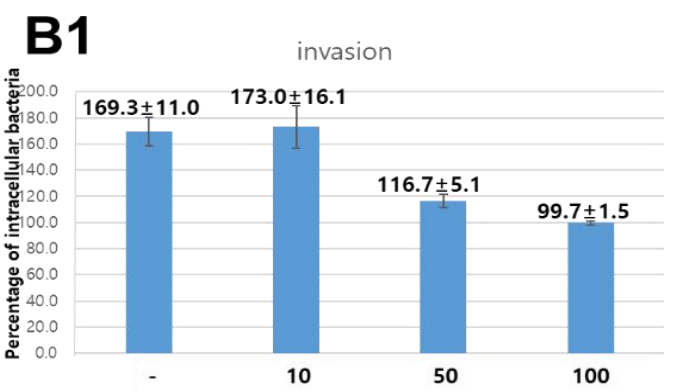

B2

\section{C1}

survival
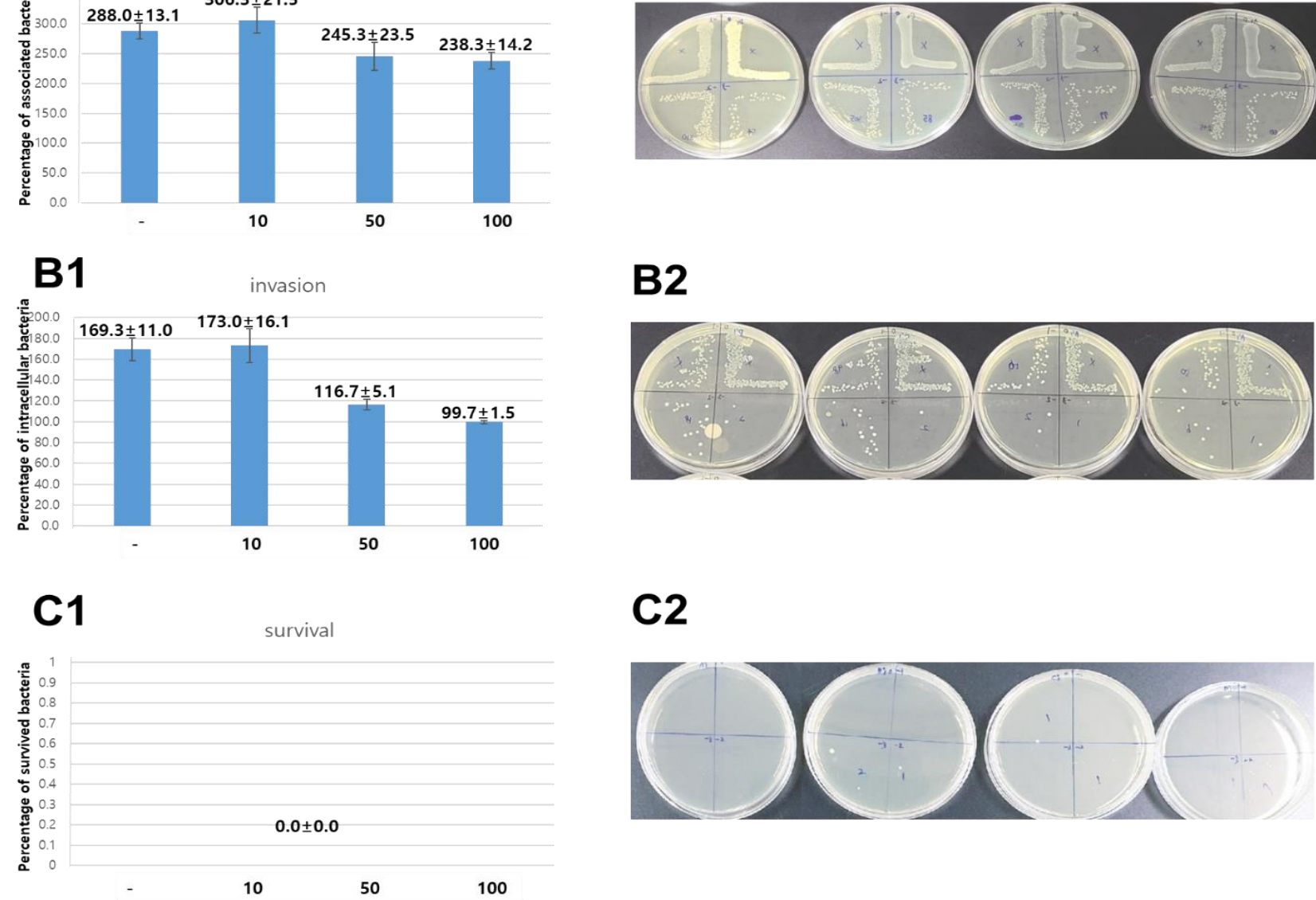

C2

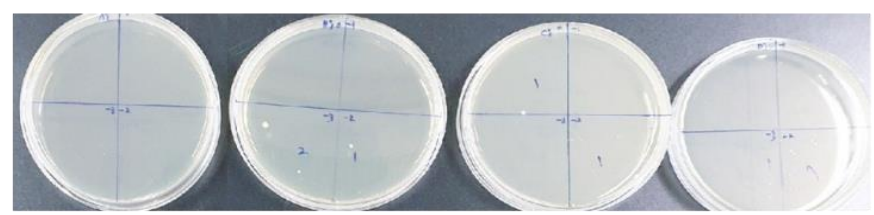

Fig. 3: S. typhi association, invasion, and survival to $N$. fowleri trophozoites. A1, B1 and C1 represented the percentage of $S$. typhi association, invasion, and survival of the bacteria, respectively, and A2, B2 and C2 represented the plates for A1, B1 and $\mathrm{C} 1$, respectively. The number on the $\mathrm{X}$-axis indicated the $\mathrm{mM}$ concentration of mannose in $\mathrm{A} 1, \mathrm{~B} 1$ and $\mathrm{C} 1$. This experiment was performed in triplicate wells with three times, and data were indicated by SD value.

S. typhi was almost $238 \%$ to $306 \%$ associated with $N$. fowleri trophozoites regardless of mannose-treated $N$. fowleri trophozoites (Fig. 3 A1, B1). On the other hand, S. typhi invasion was reduced by $52.6 \%$ in $50 \mathrm{mM}$ mannose and $69.6 \%$ in $100 \mathrm{mM}$ mannose compared to the control when mannose was treated. Very interestingly, although S. typhi showed much higher invasion than the above MRSA and E. faecalis, it did not survive at all within $N$. fowleri trophozoites.

Effect of mannose on $N$. fowleri cysts in the interaction with $S$. typhi

$N$. fowleri cysts have less motility than $N$. fowleri trophozoites, but the thickness of its cell wall is thicker than trophozoite type. However, rather than the $N$. fowleri cyst, Acanthamoeba cyst has a very thick double membrane. In this experiment, the interaction between $S$. typhi and $N$. fowleri cyst, which showed the highest association and invasion, was analyzed. As shown in Fig. 4, compared to the control group, $10 \mathrm{mM}$ mannose showed a nearly similar $1 \%$ association with $N$. fowler $i$ cyst treated, but not with $50 \mathrm{mM}$ and $100 \mathrm{mM}$ mannose treated $N$. fowleri cyst at all. On the other hand, S. typhi invasion and survival were all $0 \%$, so it was not shown in this figure.

association

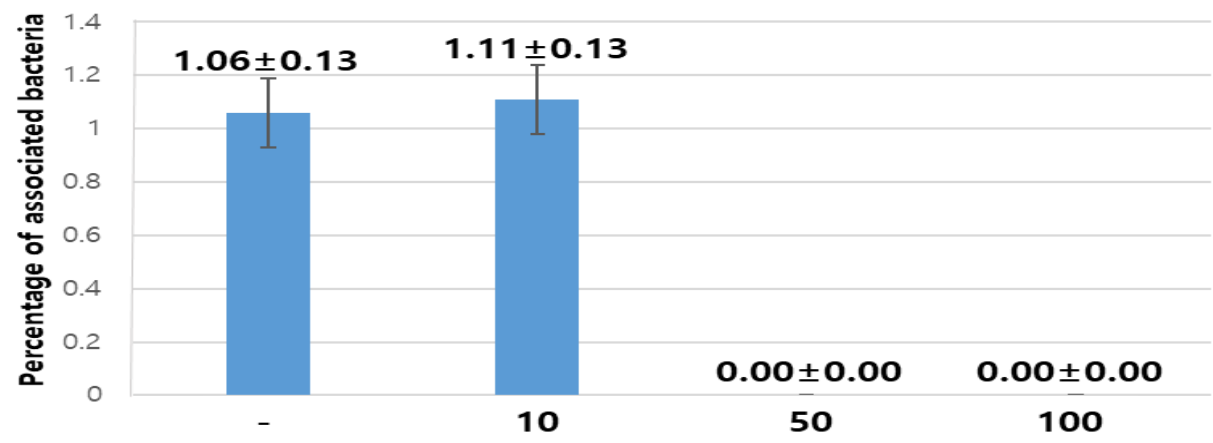

Fig. 4: S. typhi association to N. fowleri cysts. This experiment was performed in triplicate wells with three times, and data were indicated by SD value. The number on the $\mathrm{X}$-axis indicated the $\mathrm{mM}$ concentration of mannose. 


\section{DISCUSSION}

Among the free-living amoebae, Acanthamoeba, which has been well analyzed for interaction with bacteria such as E. coli, S. aureus, Pseudomonas aeruginosa, etc., could interact with various bacteria and bind to the bacterial cell wall to ingest bacteria. There is little analysis of the monosaccharide or polysaccharide effects of free-living amoebae, but one study found that highly interacting L. pneumophila was the $\alpha 1-3 \mathrm{D}-$ mannobiose domain of the mannose-of $A$. castellanii (18). Moreover, L. pneumophila has high affinity with GalNAc $\beta 1-4 \mathrm{Gal}$ domain of the N-acetyl-Dgalactosamine receptor of $N$. lovaniensis. Thus, monosaccharides or polysaccharides located outside the cell wall of bacteria would be able to bind to the glycosylated protein of Naegleria spp. In N. fowleri, there have been recent reports on the association of mannose and fucose with host cell adhesion and cytotoxicity (19). Therefore, the possibility of vaccine and diagnosis was suggested using lectin, a sugarbinding protein. In this study, it was analyzed with the association of some clinically important bacteria, e.g., MRSA, E. faecalis, $S$. typhi, with $N$. fowleri trophozoites treated with mannose, and analyzed the number of bacteria that could invade and survive after the bacteria bound with $N$. fowleri trophozoites. Furthermore, the interaction between mannose-treated $N$. fowleri cysts morphologically different from $N$. fowleri trophozoites and bacteria was also analyzed. Of the three bacteria analyzed above, the percentage of $S$. typhi interacting with $N$. fowleri trophozoites was the highest, but none of the bacteria survived within the cytoplasm of $N$. fowleri trophozoites and cysts. There was currently no report on the reason for supplementing these results, but it was thought that the bacteria entering and invading by various proteolytic enzymes in the cytoplasm of $N$. fowleri might be killed by incubating for $24 \mathrm{hr}$ for survival evaluation. There was a report that explained the survival of bacteria within Acanthamoeba and the process by which Acanthamoeba consumed bacteria. The ability of $A$. castellanii to promote the survival of Vibrio parahaemolyticus POR1 strain could be promoted by factors secreted from $A$. castellanii, not by direct contact between $A$. castellanii and $V$. parahaemolyticus POR1 (20). This can be explained as chemotaxis. When $N$. fowleri trophozoites bound to target cells, it stimulated the mobility and proliferation of $N$. fowleri trophozoites due to the selective chemotactic factor. If it was reinforced by the downstream signals, the chemotaxis could be more and more reinforced (21-22). With binding assays using different lectins, Naegleria spp. showed the abundance of surface glycoconjugates containing $\alpha$-D-glucosyl, $\alpha$ D-mannosyl and $\alpha$-L-fucosyl, N-acetyl- $\alpha$-Dgalactosaminyl and $\alpha$-D-galactose residues $(8,23)$. On the other hand, the expression of monosaccharide residues in these glycoproteins had the potential to be evaluated as an index capable of distinguishing and diagnosing pathogenic and non-pathogenic Naegleria
(8). When biotinylated lectin was used, alpha-Dmannose, alpha-D-glucose, and terminal alpha-Lfucose residues were observed to be higher in pathogenic $N$. fowleri than in non-pathogenic $N$. gruberi (8). In another report, analysis of the difference in carbohydrate content between $N$. folweri and $N$. lovaniensis showed thicker aggregates for mannose and galactose in $N$. folweri than N. lovaniensis (24).

\section{CONCLUSION}

Analysis of bacterial interactions on Naegleria is still inadequate. In this study, the association, invasion, and survival of mannose-treated $N$. fowleri trophozoites and cysts and bacteria such as MRSA, E. faecalis and $S$. typhi were analyzed using mannose, which was considered important for contact with target cells or bacteria in free-living amoebae. The association and invasion of $S$. typhi was highest for $N$. fowleri trophozoites and cysts, but the three bacteria did not survive in $N$. fowleri trophozoites and cysts. Therefore, this study suggests that mannose played an important role in association and invasion of $N$. fowleri and bacterial interactions. Therefore, this study may be helpful in analyzing how $N$. fowleri survives in the environment and its interaction with host cells.

\section{ACKNOWLEDGMENT}

Funding for this paper was provided by Namseoul University.

\section{CONFLICT OF INTEREST}

Author has no conflict of interest.

\section{REFERENCES}

1. Siddiqui, R., Ali, I. K. M., Cope, J. R., Khan, N. A. Biology and pathogenesis of Naegleria fowleri. Acta Trop. 2016; 164: 375394.

2. Jahangeer, M., Mahmood, Z., Munir, N., Waraich, U. E., Tahir, I. M., Akram, M., Ali, Shah, S. M., Zulfqar, A., Zainab, R. Naegleria fowleri: Sources of infection, pathophysiology, diagnosis, and management; a review. Clin Exp Pharmacol Physiol. 2020; 47(2): 199-212.

3. Walochnik, J, Müller, K. D., Aspöck, H., Michel, R. An endocytobiont harbouring Naegleria strain identified as $\mathrm{N}$. clarki De Jonckheere, 1994. Acta Protozool. 2005; 44(4): 301310.

4. Visvesvara, G. S., Moura, H., Schuster, F. L. Pathogenic and opportunistic free-living amoebae: Acanthamoeba spp., Balamuthia mandrillaris, Naegleria fowleri, and Sappinia diploidea. FEMS Immunol Med Microbiol. 2007; 50(1): 1-26.

5. Jamerson, M., Schmoyer, J. A., Park, J., Marciano-Cabral, F., Cabral, G. A. Identification of Naegleria fowleri proteins linked to primary amoebic meningoencephalitis. Microbiol. 2017; 163(3): 322-332.

6. Sohn, H. J., Kim, J. H., Shin, M. H., Song, K. J., Shin, H. J. The $\mathrm{Nf}$-actin gene is an important factor for food-cup formation and cytotoxicity of pathogenic Naegleria fowleri. Parasitol Res. 2010; 106(4): 917-924.

7. Pettit, D. A. D, Williamson, J., Cabral, G. A., Marciano-Cabral, F. In vitro destruction of nerve cell cultures by Acanthamoeba spp.: A transmission and scanning electron microscopy study. J Parasitol. 1996; 82(5): 769-777.

8. Cervantes-Sandoval, I., Serrano-Luna, J. J., Pacheco-Yépez, J., Silva-Olivares, A., Tsutsumi, V., Shibayama, M. Differences between Naegleria fowleri and Naegleria gruberi in expression 
of mannose and fucose glycoconjugates. Parasitol Res. 2010; 106(3): 695-701.

9. Alsam, S., Jeong, S. R., Sissons, J., Dudley, R., Kim, K. S., Khan, N. A. Escherichia coli interactions with Acanthamoeba: a symbiosis with environmental and clinical implications. J Med Microbiol. 2006; 55(6): 689-694.

10. Jung, S. Y. Free living amoeba-bacteria interactions: Analysis of Escherichia coli interactions with nonpathogenic or pathogenic free-living amoeba. J Exp Biomed Sci. 2011; 17(1): 7-12.

11. Jung, S. Y., Matin, A., Kim, K. S., Khan, N. A. The capsule plays an important role in Escherichia coli $\mathrm{K} 1$ interactions with Acanthamoeba. Int J Parasitol. 2007; 37(3-4): 417-423.

12. Rowbotham, T. J. Preliminary report on the pathogenicity of Legionella pneumophila for freshwater and soil amoebae. J Clin Pathol. 1980; 33(12): 1179-1183.

13. Young J. D. E., Lowrey, D. M. Biochemical and functional characterization of a membrane-associated pore-forming protein from the pathogenic ameboflagellate Naegleria fowleri. J Biol Chem. 1989; 264(2): 1077-1083.

14. Kang, A. Y., Park, A. Y., Shin, H. J., Khan, N. A., Maciver, S. K., Jung, S. Y. Production of a monoclonal antibody against a mannose-binding protein of Acanthamoeba culbertsoni and its localization. Exp Parasitol. 2018; 192; 19-24.

15. Jung, S. Y. Inhibition of interactions between Acanthamoeba culbertsoni trophozoites and bacteria by antibodies to a mannose-binding protein. Biomedicine. 2020; 40(2): 198-202.

16. Willaert, E. Isolement et culture in vitro des amibes de genre Naegleria. Ann Soc Belg Med Trop. 1973; 51(6): 701-708.

17. Lim, K. B., Boey, L. P., Khatijah, M. Gram's-stained microscopy in the etiological diagnosis of Malassezia (Pityrosporon) folliculitis. Arch Dermatol. 1988; 124(4): 492.

18. Declerck, P., Behets, J., Keersmaecker, B. D., Ollevier, F. Receptor-mediated uptake of Legionella pneumophila by Acanthamoeba castellanii and Naegleria lovaniensis. J Appl Microbiol. 2007; 103(6): 2697-2703.

19. Guzmán-Téllez, P., Martínez-Castillo, M., Flores-Huerta N., Rosales-Morgan, G., Pacheco-Yépez, J., de la Garza, M., et al., Lectins as virulence factors in Entamoeba histolytica and freeliving amoebae. Future Microbiol. 2020; 15(10): 919-936.

20. Laskowski-Arce, M. A., Orth, K. Acanthamoeba castellanii promotes the survival of Vibrio parahaemolyticus. Appl Environ Microbiol. 2008; 74(3): 7183-7188.

21. Baig, A. M. Primary amoebic meningoencephalitis: neurochemotaxis and neurotropic preferences of Naegleria fowleri. ACS Chem Neurosci. 2016; 7(8): 1026-1029.

22. Jahangeer, M., Mahmood, Z., Munir, N., Waraich, U., Tahir, I. M., Akram, M., et al., Naegleria fowleri: Sources of infection, pathophysiology, diagnosis, and management; a review. Clin Exp Pharmacol Physiol. 2020; 47(2): 199-212.

23. Betanzos, A., Bañuelos, C., Orozco, E. Host invasion by pathogenic amoebae: epithelial disruption by parasite proteins. Genes (Basel) 2019; 10(8): 618.

24. González-Robles, A., Castañón, G., Cristóbal-Ramos, A. R., Hernández-Ramírez, V. I., Omaña-Molina, M., MartínezPalomo. A. Cell surface differences of Naegleria fowleri and Naegleria lovaniensis exposed with surface markers. Exp Parasitol. 2007; 117(4): 399-404. 